\title{
Finding a Tandem Repeats Motifs in the Completed Genomes of Human Coronavirus (hku1) Which is Identified as a Hotspot Region for the Viruses Recombination by Using Regular Expression Language
}

Mohammed Ahmed Ezzelregal Hassan ${ }^{1}$, Mohamed Elsayed Hasan ${ }^{2}$

1. PhD. in Bioinformatics, University of SADAT City, Genetic Engineering and Biotechnology Research Institute GEBRI, Bioinformatics Department, Mohammed.ahmed.stu@gebri.usc.edu.eg

Bioinformatician and molecular geneticist at Molecular genetics and taxonomy lab, General Authority for Fish Resources and Development (GAFRD)

2. PhD. in Bioinformatics, University of SADAT City, Genetic Engineering and Biotechnology Research Institute GEBRI, Bioinformatics Department , mohamed.elsaid@gebri.usc.edu.eg

Bioinformatician at the central laboratory unit of GEBRI institute 


\section{Abstract}

A lot of research studies have been surveyed the completed genomes of prokaryotic and eukaryotic and focused on the correlation between the percentage of microsatellite sequences in completed genomes and the whole size of the organism genomes. There are fewer studies made in repetitive sequences otherwise simple sequence repeats or long tandem repeats of virus genomes. simple sequence repeats (SSRs) are the most important regions for recombination and moving repeats blocks from site to another site in the genomes. A tool was programmed and designed by visual basic 6.0 to find the long tandem repeats in DNA sequences of the small genomes. The tool named "Repeater Finder Regular Expression", (RFRE) Version 1.0, 2016. The tool was utilized to discover different pattern of long tandem repeats (LTR) motifs on the completed genomes of human corona virus strains by using a joined regular expression language. In this study, a twenty-nine accession numbers of human coronavirus completed genomes, (hku1) strains were retrieved from the Genbank. The researcher can write a different regular expression patterns and joined regular expression patterns through the designed tool to search and find a specific motif of nucleotide sequences inside the complete genomes. The RFRE tool searched and found three different total lengths of a perfect long tandem repeats (240bp, 300bp and 480bp). A Dot plot gave a picture view for the long tandem repeat sequences in the completed genome sequence (KF430201.1) of human coronavirus. The genomic dot plot tool YASS was used as a genomic similarity searching tool to check for the uninterrupted repeats and confirm the sensitivity of the (RFRE) tool. To identify the recombination site in the genomes of human coronavirus the RAT tool was applied to find the recombination sites between the completed genomes of human corona viruses. The RAT tool recognized the recombination site in the nucleotide position (3012) and at the same time this 
recombination site position (3012) was also recognized as a beginning position of a long tandem repeat. A precise motif was predicted from the translated repeats of Human Corona Virus which found by PRATT tool. There was a relationship between the total length of long tandem repeats and genome size of Human Corona Virus and the correlation value $\mathrm{R}^{2}$ was equal to $(0,451)$. In conclusion, this study presented the importance of finding the long tandem repeats of human coronavirus and gives a relationship between the completed genome size of human coronavirus types and long tandem repeats. The nucleotide position (3012) was a hot spot site for a recombination among the complete genomes of human coronavirus and also identified as a repetitive site in the genomes of human corona virus (hku1). The repeats in human coronavirus (hku1) were predicated to be a main major role of virus evolution.

Key words: Repeats Blocks; Repeater Finder Regular Expression; Tandem Repeats of Human Coronavirus

\section{Introduction}

A lot of studies had been made in the finished genomes of prokaryotic and eukaryotic to illustrate a correlation between the total length of different repeats patterns in the completed genomes and genome size but there are a small numbers of research studies are made in the completed genomes of virus to illustrate the correlation between the total length of repetitive sequences in the virus genome and genome size of viruses [1]. Twenty-two strains of human coronavirus, (HKU1) completed genomes were matched after sequencing and categorized in different genotypes (A, B and C) [2]. The human coronavirus, (HKU1) strains contain an acidic tandem repeats, which encodes the following amino acids residues - NDDEDVVTGD [2]. The recombination mechanism in human coronavirus lead to form a new four genotypes of human coronavirus ( $A$, 
$B, C$ and D) [3]. The genotypes of human coronavirus, (HKU1) linked with human infection and this linkage was responsible for new genotype of human coronavirus, (HKU1) [3]. Recombination between the repeat regions in the finished genomes of different human coronavirus, (HKU1) strains was observed and considered to be a main major role of human coronavirus evolution [2]. Imperfect microsatellites repeats are detected in the different strains of coronavirus (HKU1)thats could be found due to the incorrect replication by the viral polymerase [2]. Recombination considered as one of the procedure of virus evolution [4]. Simple sequence repeats are the essential sites for virus recombination and exchange repeats blocks from region to another region in the human genome [5]. There is a correlation between the size of viruses' genome and SSR density [1]. The great variances in genome size of viruses give a wide possible to study the perfect and imperfect repeats length of virus's genome [1]. The number of repeats was caused by an error rates of viral polymerase replication or by recombination evidence between the repeat blocks of different virus's strains [6]. A number of herpes viruses' telomeric repeats (TMRs) are identical to the telomeric repeat sequences of the host in their genomes, TMRs although they are genetically classifying herpes virus to classes A, C, D, or E genome [7]. Some SSR sequences can stimulate the recombination and special effects on DNA structure where the repeats (GT, CA, CT, GA, GC, or AT) were considered as repeat-binding proteins [8].

\section{Material and Methods}

\subsection{Retrieving (hku1) strains of coronavirus}

A twenty-nine completed genome records were retrieved from the primary database of Genbank, NCBI core nucleotide database and downloaded in fasta format file then organized in (Table 1). 
Table 1. The Retrieved Accession Numbers (twenty-nine records) of Human coronavirus Completed Genome ((HKU1)) from Archived Genbank of NCBI.

\begin{tabular}{cccc}
\hline Accession number & Genome Size & Accession number & Genome Size \\
\hline DQ415904.1 & 29295 & HM034837 & 29862 \\
KF686344.1 & 29695 & DQ415914.1 & 29887 \\
KF686342.1 & 29742 & DQ415911.1 & 29902 \\
DQ415909.1 & 29767 & DQ415903.1 & 29920 \\
DQ415913.1 & 29785 & KF686340.1 & 29923 \\
DQ415910.1 & 29797 & AY597011.2 & 29926 \\
DQ415900.1 & 29812 & NC_006577.2 & 29926 \\
DQ415902.1 & 29845 & KF430201.1 & 29934 \\
DQ415912.1 & 29845 & DQ415908.1 & 29947 \\
DQ415905.1 & 29857 & KF686346.1 & 29982 \\
DQ415906.1 & 29857 & KF686343.1 & 29983 \\
DQ415901.1 & 30097 & DQ415897.1 & 29845 \\
DQ339101.1 & 29755 & DQ415898.1 & 29815 \\
KF686341.1 & 29832 & DQ415899.1 & 29905 \\
DQ415896.1 & 29860 & & \\
\hline
\end{tabular}

\subsection{Analyzing the completed genomes}

The repeated sequences of the (hku1) strain of coronavirus completed genomes were found and analyzed by a new-programmed tool, RFRE Version 1.0 (Repeater Finder Regular Expression). The tool can be downloaded via the following link (https://sourceforge.net/projects/rfret/). The RFRE tool found the lengths of repeat, positions of repeat, frequencies of repeat and the densities of repeat. The tool used the regular expression language to find different types of patterns and the user could easily change the syntax of regular expression command to retrieve different forms of specific repeats. The tool had an easily GUI (Graphical User Interface) which could be used by a nonprofessional user. For example the regular expression pattern ([agct] $\{30\}) \backslash 1$ was accepted by the RFRE tool to find a different repeated patterns with sixty characters in length. The input pattern 
([agct]\{30\})\1 was used to match any pattern of characters a or $\mathrm{g}$ or $\mathrm{c}$ or $\mathrm{t}$ [agct] and repeated two times by a length thirty characters $\{30\}$. The Metacharcter back reference $\backslash 1$ was used to match the same text again. Back references match the same text as previously matched by a catching group, for example the same back reference can be used again more than once ([y|c])x\1 matches yxyxa, cxcxb and cxcxc. The outputs results of using RFRE tool could be saved in word file or excel sheet.

\subsection{Organizing the output of the results in excel sheets}

The results were archived and recorded in the excel sheet to finalize the results in charts to show the relationship between the genome size and the repeat length.

\subsection{Predict a specific motif for the extracted repeats}

All the extracted perfect repeats were aligned by using MAFFT tool, which was used as a plugin of Unipro UGENE version (1.16.1, Mar 17-2015) to find a highly conserved sequence. The conserved sequence was saved in a fasta format file and converted to a protein sequence by using ORF finder tool (http://www.ncbi.nlm.nih.gov/projects/gorf/). The translated frame was used to hit the protein sequence database by using PSI-BLAST of Primary database of Genbank, European bioinformatics institute (EBI) which had the following web address (http://www.ebi.ac.uk/Tools/sss/psiblast). EBI had The PRATT tool, (http://www.ebi.ac.uk/Tools/pfa/pratt/) which was used to search for a conserved patterns in sets of aligned protein sequences, which aligned to the translated pattern of the conserved perfect repeat. The scanprosite of Expasy, bioinformatics resource portal server (http://prosite.expasy.org/scanprosite/) 
was fed by the expected pattern which produced by PRATT tool to find a specific motif in the Human coronavirus (hku1).

\subsection{Using the Dot plot to present the repeats in a picture view}

To find a picture view for a repeated sequences in the completed genome sequence (KF430201.1) of human coronavirus the YASS online tool (http://bioinfo.lifl.fr/yass) was used to produce this picture view in dot plot view. YASS is a genomic similarity search tool to produce a local alignment results. YASS, Like most of the heuristic pairwise local alignment tools for DNA sequences (FASTA, BLAST, PATTERNHUNTER, BLASTZ/LASTZ, LAST).

\subsection{Detecting recombination among genomes}

The RAT (Recombination analysis tool) program was used to detect the recombination between multiple sequences, in a straightforward graphical user interface. The fasta format of the human corona virus completed genome were downloaded and organized in the previous Table, (Table1) then aligned by clustalw omega (www.ebi.ac.uk/Tools/msa/clustalo/) The RAT tool processed the multiple aligned sequences. The RAT tool was downloaded from this web site (https://github.com/ethering/RAT). The result can be exported to excel file included the possible recombination spots.

\subsection{Assign the repeats to annotated map}

The highest total length of the extracted repeats which found in the completed genome of Human corona virus (kf686343.1) were assigned to the annotated map of the following accession number (kf686343.1) by using Unipro UGENE ver. $1.16,2015$ by download the Genbank report of the following accession number (kf686343.1). 


\section{Results and discussion}

\subsection{The input regular expression and back references}

To find repeated sequences in twenty nine records of human coronavirus completed genome a specific regular expression command was used, $([a g c t]\{30\}) \backslash 1$ through the designed tool (RFRE 1.0) which accepted that regular expression behavior. The behavior of using the regular expression, $([a g c t]\{30\}) \backslash 1$ found a repeated sequences in groups and in a perfect form. The meaning of every metacharacter in the previous regular expression, the metacharacter () was meant it matched a pattern and captured the matched pattern where the captured match can be retrieved from the resulting matched collection. Metacharacter [agct] was meant it matched a or $\mathrm{g}$ or $\mathrm{c}$ or $\mathrm{t}$ and the metacharacter $\{30\}$ was meant it matched exactly $\mathrm{n}$ times but the memory parentheses and back references was used $\backslash 1$ to make the regular sub expression in parentheses $(r)$, the matched subsequence was remembered. The matched subsequence was bound to the matched variable $\backslash 1$. If there were more parentheses, their matched get bound to 12,13 , etc. so the previous regular expression found the pattern more than one period and the length of the found repeats were 60 characters in length. A simple example for the backreference system, A repeat such as ACTACT in a DNA sequence can be described by $([A C G T]+) \backslash 1$ The nucleotide sequence matched [ACGT]+ must be appeared again to be matched by $\backslash 1$. The repeats, which were found were organized and described in (Table 2). A comparison between the total of repeat length and genome size in different strains of human corona virus was 
presented that the following accession numbers (kf686346.1 and kf686343.1) had the same highest repeat length(480 bp) which bolded and shaded by gray color as shown in the (Table 2 \& Table 3). All 22 genomes of Coronavirus (HKU1) Genomes had tandem duplicates of a perfect 30-base acidic tandem repeat (ATR) which encodes NDDEDVVTGD [2]. The (Fig.1) displayed the comparison between the total of repeat lengths and genome size in different strains of human corona virus. There were a fluctuation among repeat lengths and genome size as shown in (Fig. 1). A correlation between the total length of repeats and genome size presented by the regression type, power of trendline as shown in (Fig. 2). The $R^{2}$ value was displayed on the chart where the $\left(R^{2}=\right.$ 0.451) as shown in (Fig. 2). A relationship between simple sequence repeats (SSRs) and virus genome size where SSRs were accumulated in virus with large genomes, and the longer genome sequence contained the longer repeat units [9].

Table 2. The results of tandem repeats which were found by using a specific regular expression syntax ([agct] $\{30\}) \backslash 1$ which written through the RFRE tool.

\begin{tabular}{|c|c|c|c|c|c|c|c|}
\hline Genome ID & Motif & Iteration & Start & End & $\begin{array}{c}\text { Repeat } \\
\text { Unit / } \\
\text { Position }\end{array}$ & $\begin{array}{l}\text { Repeat } \\
\text { Length }\end{array}$ & $\begin{array}{c}\text { Genome } \\
\text { Size }\end{array}$ \\
\hline DQ415910.1 & aatgacgatgaagatgttgttactggtgac & 2 & 3031 & 3091 & 30 & 60 & 29797 \\
\hline DQ415910.1 & aatgacgatgaagatgttgttactggtgac & 2 & 3091 & 3151 & 30 & 60 & \\
\hline DQ415910.1 & aatgacgatgaagatgttgttactggtgac & 2 & 3151 & 3211 & 30 & 60 & \\
\hline DQ415910.1 & aatgacgatgaagatgttgttactggtgac & 2 & 3211 & 3271 & 30 & 60 & \\
\hline DQ415910.1 & aatgacgatgaagatgttgttactggtgac & 2 & 3271 & 3331 & 30 & 60 & \\
\hline DQ415909.1 & aatgacgatgaagatgttgttactggtgac & 2 & 3031 & 3091 & 30 & 60 & 29767 \\
\hline DQ415909.1 & aatgacgatgaagatgttgttactggtgac & 2 & 3091 & 3151 & 30 & 60 & \\
\hline DQ415909.1 & aatgacgatgaagatgttgttactggtgac & 2 & 3151 & 3211 & 30 & 60 & \\
\hline DQ415909.1 & aatgacgatgaagatgttgttactggtgac & 2 & 3211 & 3271 & 30 & 60 & \\
\hline DQ415908.1 & aatgacgatgaagatgttgttactggtgac & 2 & 3031 & 3091 & 30 & 60 & 29947 \\
\hline$\overline{D Q 415908.1}$ & aatgacgatgaagatgttgttactggtgac & 2 & 3091 & 3151 & 30 & 60 & \\
\hline DQ415908.1 & aatgacgatgaagatgttgttactggtgac & 2 & 3151 & 3211 & 30 & 60 & \\
\hline DQ415908.1 & aatgacgatgaagatgttgttactggtgac & 2 & 3211 & 3271 & 30 & 60 & \\
\hline DQ415908.1 & aatgacgatgaagatgttgttactggtgac & 2 & 3271 & 3331 & 30 & 60 & \\
\hline DQ415908.1 & aatgacgatgaagatgttgttactggtgac & 2 & 3331 & 3391 & 30 & 60 & \\
\hline DQ415908.1 & aatgacgatgaagatgttgttactggtgac & 2 & 3391 & 3451 & 30 & 60 & \\
\hline
\end{tabular}




\begin{tabular}{|c|c|c|c|c|c|c|c|}
\hline DQ415906.1 & aatgacgatgaagatgttgttactggtgac & 2 & 3031 & 3091 & 30 & 60 & 29857 \\
\hline DQ415906.1 & aatgacgatgaagatgttgttactggtgac & 2 & 3091 & 3151 & 30 & 60 & \\
\hline DQ415906.1 & aatgacgatgaagatgttgttactggtgac & 2 & 3151 & 3211 & 30 & 60 & \\
\hline DQ415906.1 & aatgacgatgaagatgttgttactggtgac & 2 & 3211 & 3271 & 30 & 60 & \\
\hline DQ415906.1 & aatgacgatgaagatgttgttactggtgac & 2 & 3271 & 3331 & 30 & 60 & \\
\hline DQ415906.1 & aatgacgatgaagatgttgttactggtgac & 2 & 3331 & 3391 & 30 & 60 & \\
\hline DQ415905.1 & aatgacgatgaagatgttgttactggtgac & 2 & 3031 & 3091 & 30 & 60 & 29857 \\
\hline DQ415905.1 & aatgacgatgaagatgttgttactggtgac & 2 & 3091 & 3151 & 30 & 60 & \\
\hline DQ415905.1 & aatgacgatgaagatgttgttactggtgac & 2 & 3151 & 3211 & 30 & 60 & \\
\hline DQ415905.1 & aatgacgatgaagatgttgttactggtgac & 2 & 3211 & 3271 & 30 & 60 & \\
\hline DQ415905.1 & aatgacgatgaagatgttgttactggtgac & 2 & 3271 & 3331 & 30 & 60 & \\
\hline DQ415905.1 & aatgacgatgaagatgttgttactggtgac & 2 & 3331 & 3391 & 30 & 60 & \\
\hline DQ415904.1 & aatgacgatgaagatgttgttactggtgac & 2 & 3031 & 3091 & 30 & 60 & 29295 \\
\hline DQ415903.1 & aatgacgatgaagatgttgttactggtgac & 2 & 3331 & 3391 & 30 & 60 & 29920 \\
\hline DQ415903.1 & aatgacgatgaagatgttgttactggtgac & 2 & 3391 & 3451 & 30 & 60 & 29920 \\
\hline DQ415902.1 & aatgacgatgaagatgttgttactggtgac & 2 & 3031 & 3091 & 30 & 60 & 29845 \\
\hline DQ415902.1 & aatgacgatgaagatgttgttactggtgac & 2 & 3091 & 3151 & 30 & 60 & \\
\hline DQ415902.1 & aatgacgatgaagatgttgttactggtgac & 2 & 3151 & 3211 & 30 & 60 & \\
\hline DQ415902.1 & aatgacgatgaagatgttgttactggtgac & 2 & 3211 & 3271 & 30 & 60 & \\
\hline DQ415902.1 & aatgacgatgaagatgttgttactggtgac & 2 & 3271 & 3331 & 30 & 60 & \\
\hline DQ415902.1 & aatgacgatgaagatgttgttactggtgac & 2 & 3331 & 3391 & 30 & 60 & \\
\hline DQ415901.1 & aatgacgatgaagatgttgttactggtgac & 2 & 3404 & 3464 & 30 & 60 & 30097 \\
\hline DQ415901.1 & aatgacgatgaagatgttgttactggtgac & 2 & 3464 & 3524 & 30 & 60 & \\
\hline DQ415901.1 & aatgacgatgaagatgttgttactggtgac & 2 & 3524 & 3584 & 30 & 60 & \\
\hline DQ415900.1 & aatgacgatgaagatgttgttactggtgac & 2 & 3031 & 3091 & 30 & 60 & 29812 \\
\hline DQ415900.1 & aatgacgatgaagatgttgttactggtgac & 2 & 3091 & 3151 & 30 & 60 & \\
\hline DQ415900.1 & aatgacgatgaagatgttgttactggtgac & 2 & 3151 & 3211 & 30 & 60 & \\
\hline DQ415900.1 & aatgacgatgaagatgttgttactggtgac & 2 & 3211 & 3271 & 30 & 60 & \\
\hline DQ415900.1 & aatgacgatgaagatgttgttactggtgac & 2 & 3271 & 3331 & 30 & 60 & \\
\hline DQ339101.1 & caatgacgatgaagatgttgttactggtga & 2 & 3030 & 3090 & 30 & 60 & 29755 \\
\hline DQ339101.1 & caatgacgatgaagatgttgttactggtga & 2 & 3090 & 3150 & 30 & 60 & \\
\hline DQ339101.1 & caatgacgatgaagatgttgttactggtga & 2 & 3150 & 3210 & 30 & 60 & \\
\hline DQ339101.1 & acgatgaagatgttgttactggtgacaata & 2 & 3245 & 3305 & 30 & 60 & \\
\hline KF686346.1 & aatgacgatgaagatgttgttactggtgac & 2 & $\underline{3012}$ & 3072 & 30 & 60 & 29982 \\
\hline KF686346.1 & aatgacgatgaagatgttgttactggtgac & 2 & 3072 & 3132 & 30 & 60 & \\
\hline KF686346.1 & aatgacgatgaagatgttgttactggtgac & 2 & 3132 & 3192 & 30 & 60 & \\
\hline KF686346.1 & aatgacgatgaagatgttgttactggtgac & 2 & 3192 & 3252 & 30 & 60 & \\
\hline KF686346.1 & aatgacgatgaagatgttgttactggtgac & 2 & 3252 & 3312 & 30 & 60 & \\
\hline KF686346.1 & aatgacgatgaagatgttgttactggtgac & 2 & 3312 & 3372 & 30 & 60 & \\
\hline KF686346.1 & aatgacgatgaagatgttgttactggtgac & 2 & 3372 & 3432 & 30 & 60 & \\
\hline KF686346.1 & aatgacgatgaagatgttgttactggtgac & 2 & 3432 & $\underline{3492}$ & 30 & 60 & \\
\hline KF430201.1 & aatgacgatgaagatgttgttactggtgac & 2 & 3027 & 3087 & 30 & 60 & 29934 \\
\hline KF430201.1 & aatgacgatgaagatgttgttactggtgac & 2 & 3087 & 3147 & 30 & 60 & \\
\hline KF430201.1 & aatgacgatgaagatgttgttactggtgac & 2 & 3147 & 3207 & 30 & 60 & \\
\hline KF430201.1 & aatgacgatgaagatgttgttactggtgac & 2 & 3207 & 3267 & 30 & 60 & \\
\hline KF430201.1 & aatgacgatgaagatgttgttactggtgac & 2 & 3267 & 3327 & 30 & 60 & \\
\hline
\end{tabular}




\begin{tabular}{|c|c|c|c|c|c|c|c|}
\hline KF430201.1 & aatgacgatgaagatgttgttactggtgac & 2 & 3327 & 3387 & 30 & 60 & \\
\hline KF430201.1 & aatgacgatgaagatgttgttactggtgac & 2 & 3387 & 3447 & 30 & 60 & \\
\hline KF686344.1 & aatgacgatgaagatgttgttactggtgac & 2 & 3012 & 3072 & 30 & 60 & 29695 \\
\hline KF686344.1 & aatgacgatgaagatgttgttactggtgac & 2 & 3072 & 3132 & 30 & 60 & \\
\hline KF686344.1 & aatgacgatgaagatgttgttactggtgac & 2 & 3132 & 3192 & 30 & 60 & \\
\hline KF686344.1 & aatgacgatgaagatgttgttactggtgac & 2 & 3192 & 3252 & 30 & 60 & \\
\hline KF686343.1 & aatgacgatgaagatgttgttactggtgac & 2 & 3012 & 3072 & 30 & 60 & 29983 \\
\hline KF686343.1 & aatgacgatgaagatgttgttactggtgac & 2 & 3072 & 3132 & 30 & 60 & \\
\hline KF686343.1 & aatgacgatgaagatgttgttactggtgac & 2 & 3132 & 3192 & 30 & 60 & \\
\hline KF686343.1 & aatgacgatgaagatgttgttactggtgac & 2 & 3192 & 3252 & 30 & 60 & \\
\hline KF686343.1 & aatgacgatgaagatgttgttactggtgac & 2 & 3252 & 3312 & 30 & 60 & \\
\hline KF686343.1 & aatgacgatgaagatgttgttactggtgac & 2 & 3312 & 3372 & 30 & 60 & \\
\hline KF686343.1 & aatgacgatgaagatgttgttactggtgac & 2 & 3372 & 3432 & 30 & 60 & \\
\hline KF686343.1 & aatgacgatgaagatgttgttactggtgac & 2 & 3432 & 3492 & 30 & 60 & \\
\hline KF686342.1 & aatgacgatgaagatgttgttactggtgac & 2 & 3012 & 3072 & 30 & 60 & 29742 \\
\hline KF686342.1 & aatgacgatgaagatgttgttactggtgac & 2 & 3072 & 3132 & 30 & 60 & \\
\hline KF686342.1 & aatgacgatgaagatgttgttactggtgac & 2 & 3132 & 3192 & 30 & 60 & \\
\hline KF686342.1 & aatgacgatgaagatgttgttactggtgac & 2 & 3192 & 3252 & 30 & 60 & \\
\hline KF686341.1 & aatgacgatgaagatgttgttactggtgac & 2 & 3012 & 3072 & 30 & 60 & 29832 \\
\hline KF686341.1 & aatgacgatgaagatgttgttactggtgac & 2 & 3072 & 3132 & 30 & 60 & \\
\hline KF686341.1 & aatgacgatgaagatgttgttactggtgac & 2 & 3132 & 3192 & 30 & 60 & \\
\hline KF686341.1 & aatgacgatgaagatgttgttactggtgac & 2 & 3192 & 3252 & 30 & 60 & \\
\hline KF686341.1 & aatgacgatgaagatgttgttactggtgac & 2 & 3252 & 3312 & 30 & 60 & \\
\hline KF686341.1 & aatgacgatgaagatgttgttactggtgac & 2 & 3312 & 3372 & 30 & 60 & \\
\hline KF686340.1 & aatgacgatgaagatgttgttactggtgac & 2 & 3012 & 3072 & 30 & 60 & 29923 \\
\hline KF686340.1 & aatgacgatgaagatgttgttactggtgac & 2 & 3072 & 3132 & 30 & 60 & \\
\hline KF686340.1 & aatgacgatgaagatgttgttactggtgac & 2 & 3132 & 3192 & 30 & 60 & \\
\hline KF686340.1 & aatgacgatgaagatgttgttactggtgac & 2 & 3192 & 3252 & 30 & 60 & \\
\hline KF686340.1 & aatgacgatgaagatgttgttactggtgac & 2 & 3252 & 3312 & 30 & 60 & \\
\hline KF686340.1 & aatgacgatgaagatgttgttactggtgac & 2 & 3312 & 3372 & 30 & 60 & \\
\hline KF686340.1 & aatgacgatgaagatgttgttactggtgac & 2 & 3372 & 3432 & 30 & 60 & \\
\hline NC_006577.2 & aatgacgatgaagatgttgttactggtgac & 2 & 3037 & 3097 & 30 & 60 & 29926 \\
\hline NC_006577.2 & aatgacgatgaagatgttgttactggtgac & 2 & 3097 & 3157 & 30 & 60 & \\
\hline NC_006577.2 & aatgacgatgaagatgttgttactggtgac & 2 & 3157 & 3217 & 30 & 60 & \\
\hline NC_006577.2 & aatgacgatgaagatgttgttactggtgac & 2 & 3217 & 3277 & 30 & 60 & \\
\hline NC_006577.2 & aatgacgatgaagatgttgttactggtgac & 2 & 3277 & 3337 & 30 & 60 & \\
\hline NC_006577.2 & aatgacgatgaagatgttgttactggtgac & 2 & 3337 & 3397 & 30 & 60 & \\
\hline NC_006577.2 & aatgacgatgaagatgttgttactggtgac & 2 & 3397 & 3457 & 30 & 60 & \\
\hline AY597011.2 & aatgacgatgaagatgttgttactggtgac & 2 & 3037 & 3097 & 30 & 60 & 29926 \\
\hline AY597011.2 & aatgacgatgaagatgttgttactggtgac & 2 & 3097 & 3157 & 30 & 60 & \\
\hline AY597011.2 & aatgacgatgaagatgttgttactggtgac & 2 & 3157 & 3217 & 30 & 60 & \\
\hline AY597011.2 & aatgacgatgaagatgttgttactggtgac & 2 & 3217 & 3277 & 30 & 60 & \\
\hline AY597011.2 & aatgacgatgaagatgttgttactggtgac & 2 & 3277 & 3337 & 30 & 60 & \\
\hline AY597011.2 & aatgacgatgaagatgttgttactggtgac & 2 & 3337 & 3397 & 30 & 60 & \\
\hline AY597011.2 & aatgacgatgaagatgttgttactggtgac & 2 & 3397 & 3457 & 30 & 60 & \\
\hline HM034837 & aatgacgatgaagatgttgttactggtgac & 2 & 3001 & 3061 & 30 & 60 & 29862 \\
\hline
\end{tabular}




\begin{tabular}{|c|c|c|c|c|c|c|c|}
\hline HM034837 & aatgacgatgaagatgttgttactggtgac & 2 & 3061 & 3121 & 30 & 60 & \\
\hline HM034837 & aatgacgatgaagatgttgttactggtgac & 2 & 3121 & 3181 & 30 & 60 & \\
\hline HM034837 & aatgacgatgaagatgttgttactggtgac & 2 & 3181 & 3241 & 30 & 60 & \\
\hline HM034837 & aatgacgatgaagatgttgttactggtgac & 2 & 3241 & 3301 & 30 & 60 & \\
\hline HM034837 & aatgacgatgaagatgttgttactggtgac & 2 & 3301 & 3361 & 30 & 60 & \\
\hline HM034837 & aatgacgatgaagatgttgttactggtgac & 2 & 3361 & 3421 & 30 & 60 & \\
\hline DQ415914.1 & aatgacgatgaagatgttgttactggtgac & 2 & 3031 & 3091 & 30 & 60 & 29887 \\
\hline DQ415914.1 & aatgacgatgaagatgttgttactggtgac & 2 & 3091 & 3151 & 30 & 60 & \\
\hline DQ415914.1 & aatgacgatgaagatgttgttactggtgac & 2 & 3151 & 3211 & 30 & 60 & \\
\hline DQ415914.1 & aatgacgatgaagatgttgttactggtgac & 2 & 3211 & 3271 & 30 & 60 & \\
\hline DQ415914.1 & aatgacgatgaagatgttgttactggtgac & 2 & 3271 & 3331 & 30 & 60 & \\
\hline DQ415914.1 & aatgacgatgaagatgttgttactggtgac & 2 & 3331 & 3391 & 30 & 60 & \\
\hline DQ415913.1 & aatgacgatgaagatgttgttactggtgac & 2 & 3031 & 3091 & 30 & 60 & 29785 \\
\hline DQ415913.1 & aatgacgatgaagatgttgttactggtgac & 2 & 3091 & 3151 & 30 & 60 & \\
\hline DQ415913.1 & aatgacgatgaagatgttgttactggtgac & 2 & 3151 & 3211 & 30 & 60 & \\
\hline DQ415913.1 & aatgacgatgaagatgttgttactggtgac & 2 & 3211 & 3271 & 30 & 60 & \\
\hline DQ415912.1 & aatgacgatgaagatgttgttactggtgac & 2 & 3031 & 3091 & 30 & 60 & 29845 \\
\hline DQ415912.1 & aatgacgatgaagatgttgttactggtgac & 2 & 3097 & 3157 & 30 & 60 & \\
\hline DQ415912.1 & aatgacgatgaagatgttgttactggtgac & 2 & 3217 & 3277 & 30 & 60 & \\
\hline DQ415912.1 & aatgacgatgaagatgttgttactggtgac & 2 & 3305 & 3365 & 30 & 60 & \\
\hline DQ415911.1 & aatgacgatgaagatgttgttactggtgac & 2 & 3031 & 3091 & 30 & 60 & 29902 \\
\hline DQ415911.1 & aatgacgatgaagatgttgttactggtgac & 2 & 3091 & 3151 & 30 & 60 & \\
\hline DQ415911.1 & aatgacgatgaagatgttgttactggtgac & 2 & 3151 & 3211 & 30 & 60 & \\
\hline DQ415911.1 & aatgacgatgaagatgttgttactggtgac & 2 & 3211 & 3271 & 30 & 60 & \\
\hline DQ415911.1 & aatgacgatgaagatgttgttactggtgac & 2 & 3271 & 3331 & 30 & 60 & \\
\hline DQ415911.1 & aatgacgatgaagatgttgttactggtgac & 2 & 3331 & 3391 & 30 & 60 & \\
\hline DQ415911.1 & aatgacgatgaagatgttgttactggtgac & 2 & 3391 & 3451 & 30 & 60 & \\
\hline$\underline{\text { AY884001.1 }}$ & gatgaagatgttgttactggtgacaatgac & 2 & 3037 & 3097 & 30 & 60 & \\
\hline AY884001.1 & gatgaagatgttgttactggtgacaatgac & 2 & 3097 & 3157 & 30 & 60 & \\
\hline AY884001.1 & gatgaagatgttgttactggtgacaatgac & 2 & 3181 & 3241 & 30 & 60 & \\
\hline AY884001.1 & gatgaagatgttgttactggtgacaatgac & 2 & 3241 & 3301 & 30 & 60 & \\
\hline AY884001.1 & gatgaagatgttgttactggtgacaatgac & 2 & 3301 & 3361 & 30 & 60 & \\
\hline DQ415896.1 & aatgacgatgaagatgttgttactggtgac & 2 & 3031 & 3091 & 30 & 60 & 29860 \\
\hline DQ415896.1 & aatgacgatgaagatgttgttactggtgac & 2 & 3091 & 3151 & 30 & 60 & \\
\hline DQ415896.1 & aatgacgatgaagatgttgttactggtgac & 2 & 3151 & 3211 & 30 & 60 & \\
\hline DQ415896.1 & aatgacgatgaagatgttgttactggtgac & 2 & 3211 & 3271 & 30 & 60 & \\
\hline DQ415896.1 & aatgacgatgaagatgttgttactggtgac & 2 & 3271 & 3331 & 30 & 60 & \\
\hline DQ415897.1 & aatgacgatgaagatgttgttactggtgac & 2 & 3031 & 3091 & 30 & 60 & 29845 \\
\hline DQ415897.1 & aatgacgatgaagatgttgttactggtgac & 2 & 3091 & 3151 & 30 & 60 & \\
\hline DQ415897.1 & aatgacgatgaagatgttgttactggtgac & 2 & 3151 & 3211 & 30 & 60 & \\
\hline DQ415897.1 & aatgacgatgaagatgttgttactggtgac & 2 & 3217 & 3277 & 30 & 60 & \\
\hline DQ415897.1 & aatgacgatgaagatgttgttactggtgac & 2 & 3305 & 3365 & 30 & 60 & \\
\hline DQ415898.1 & aatgacgatgaagatgttgttactggtgac & 2 & 3031 & 3091 & 30 & 60 & 29815 \\
\hline DQ415898.1 & aatgacgatgaagatgttgttactggtgac & 2 & 3091 & 3151 & 30 & 60 & \\
\hline DQ415898.1 & aatgacgatgaagatgttgttactggtgac & 2 & 3151 & 3211 & 30 & 60 & \\
\hline DQ415898.1 & aatgacgatgaagatgttgttactggtgac & 2 & 3211 & 3271 & 30 & 60 & \\
\hline
\end{tabular}




\begin{tabular}{llllllll} 
DQ415898.1 & aatgacgatgaagatgttgttactggtgac & 2 & 3271 & 3331 & 30 & 60 & \\
DQ415899.1 & aatgacgatgaagatgttgttactggtgac & 2 & 3031 & 3091 & 30 & 60 & $\mathbf{2 9 9 0 5}$ \\
DQ415899.1 & aatgacgatgaagatgttgttactggtgac & 2 & 3091 & 3151 & 30 & 60 & \\
DQ415899.1 & aatgacgatgaagatgttgttactggtgac & 2 & 3151 & 3211 & 30 & 60 & \\
DQ415899.1 & aatgacgatgaagatgttgttactggtgac & 2 & 3211 & 3271 & 30 & 60 & \\
DQ415899.1 & aatgacgatgaagatgttgttactggtgac & 2 & 3271 & 3331 & 30 & 60 & \\
DQ415899.1 & aatgacgatgaagatgttgttactggtgac & 2 & 3331 & 3391 & 30 & 60 & \\
DQ415899.1 & aatgacgatgaagatgttgttactggtgac & 2 & 3395 & 3455 & 30 & 60 & \\
\hline
\end{tabular}

Table 3.0 Conclusion Table for the total length of repeats and Genome Size.

\begin{tabular}{lcc}
\hline Accession Number & Genome Size & Total Length of Repeat \\
\hline DQ415904.1 & 29295 & 60 \\
kf686344.1 & 29695 & 240 \\
KF686342.1 & 29742 & 240 \\
DQ415909.1 & 29767 & 240 \\
DQ415913.1 & 29785 & 240 \\
DQ415910.1 & 29797 & 300 \\
DQ415900 & 29812 & 300 \\
DQ415902.1 & 29845 & 360 \\
DQ415912.1 & 29845 & 240 \\
DQ415905.1 & 29857 & 360 \\
DQ415906 & 29857 & 360 \\
HM034837 & 29862 & 420 \\
DQ415914.1 & 29887 & 360 \\
DQ415911.1 & 29902 & 420 \\
DQ415903.1 & 29920 & 120 \\
KF686340.1 & 29923 & 420 \\
AY597011.2 & 29926 & 420 \\
NC_006577.2 & 29926 & 420 \\
kf430201.1 & 29934 & 420 \\
DQ415908.1 & 29947 & 420 \\
kf686346.1 & 29982 & 480 \\
kf686343.1 & 29983 & 480 \\
DQ415901 & 30097 & 180 \\
DQ339101.1 & 29755 & 240 \\
KF686341.1 & 29832 & 360 \\
DQ415896.1 & 29860 & 300 \\
DQ415897.1 & 29845 & 300 \\
DQ415898.1 & 29815 & 300 \\
DQ415899.1 & 29905 & \\
AY884001.1 & 29815 & \\
\hline & & 300 \\
\hline
\end{tabular}




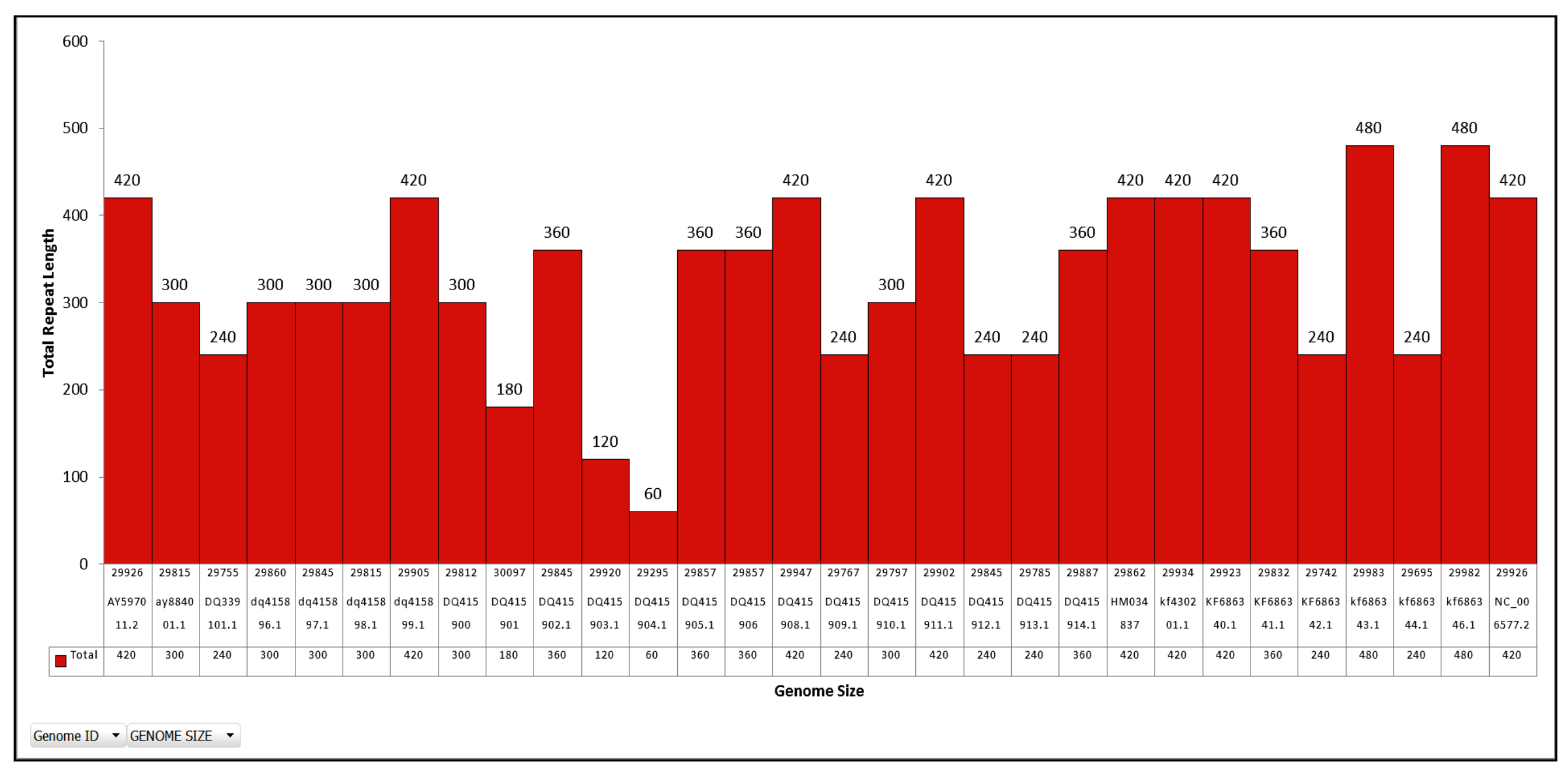

(Fig. 1) The comparison between the total length of repeats and genome size in different completed genomes of (CoV(HKU1)).

(4)




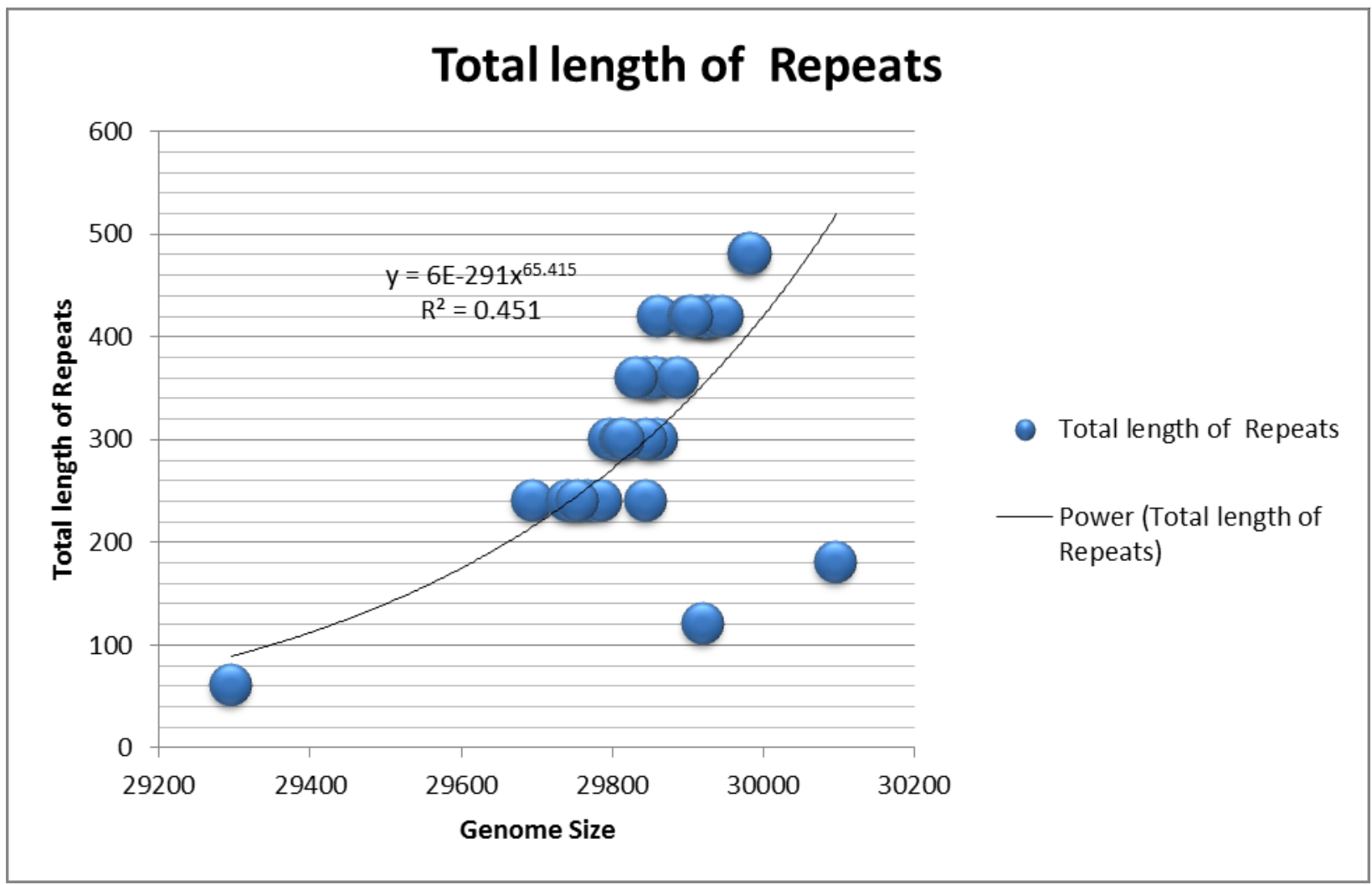

(Fig 2.) A correlation between the total length of repeats and genome size of Human Corona Virus (CoV(HKU1)).

\subsection{A specific motif was predict from the extracted repeats of Human Corona Virus}

All the previous extracted perfect repeats of twenty nine records of CoV(HKU1) were aligned with MAFFT tool which was a built module in the package of Unipro UGENE VERSION 1.16 and produced a highly conserved block which was ranged from position (181 - 240) as shown in (Fig. 3). ORF finder tool of NCBI gave the six forward frames of the submitted query sequence(conserved position,181-240) and the selected frame which was used was (ORF:1..240 Frame +1) where the length of the translated repeat was sixty nine amino acid (DDEDVVTGDNDDEDVVTGDNDDEDVVTGDNDDEDVVTGDNDDEDVVTGDNDD EDVVTGDNDDEDVVT). The selected frame of translation (ORF:1..240 Frame +1) 
was used as a seed to hit the protein sequence database by using PSI-BLAST of Primary database of Genbank- European bioinformatics institute (EBI) (http://www.ebi.ac.uk/Tools/sss/psiblast/) to produce significant pair wise alignment with thirty nine targeted sequences [Human coronavirus (HKU1)]. The aligned sequences were downloaded as a fasta format file and pasted in the input textbox of the PRATT tool (http://www.ebi.ac.uk/Tools/pfa/pratt/) which produced the best pattern (V-V-T-G-D-N-D-D-E-D-V-V-T-G-D-N-D-D-E-D). The expected pattern had a fitness score (83.4010). The shaded alignment of hits presented in clustalw format of alignment was produced by scanprosite search engine (prosite.expasy.org/scanprosite) as shown in Table 4. The shaded alignment of hits gave fifty-four hits (by the best pattern) on six sequences of human coronavirus as shown in Table 4.

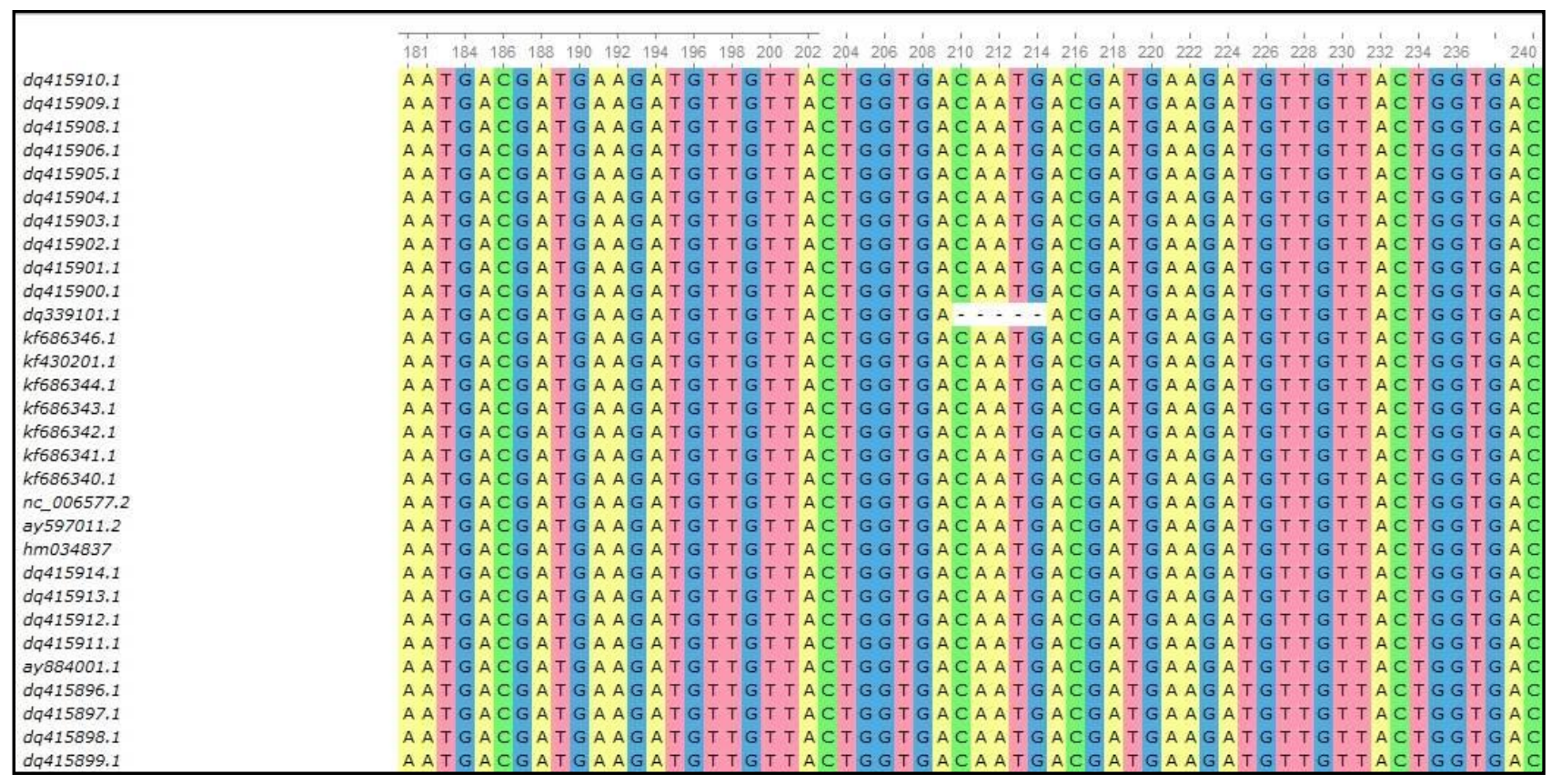

(Fig 3.) The consensus repeated sequences which detected by the RFRE tool. The consensus position (181 - 240) which located in 29 records of completed genome of human coronavirus. 
Table 4.0 Shown 54 hits (by 1 pattern) on 6 sequences.

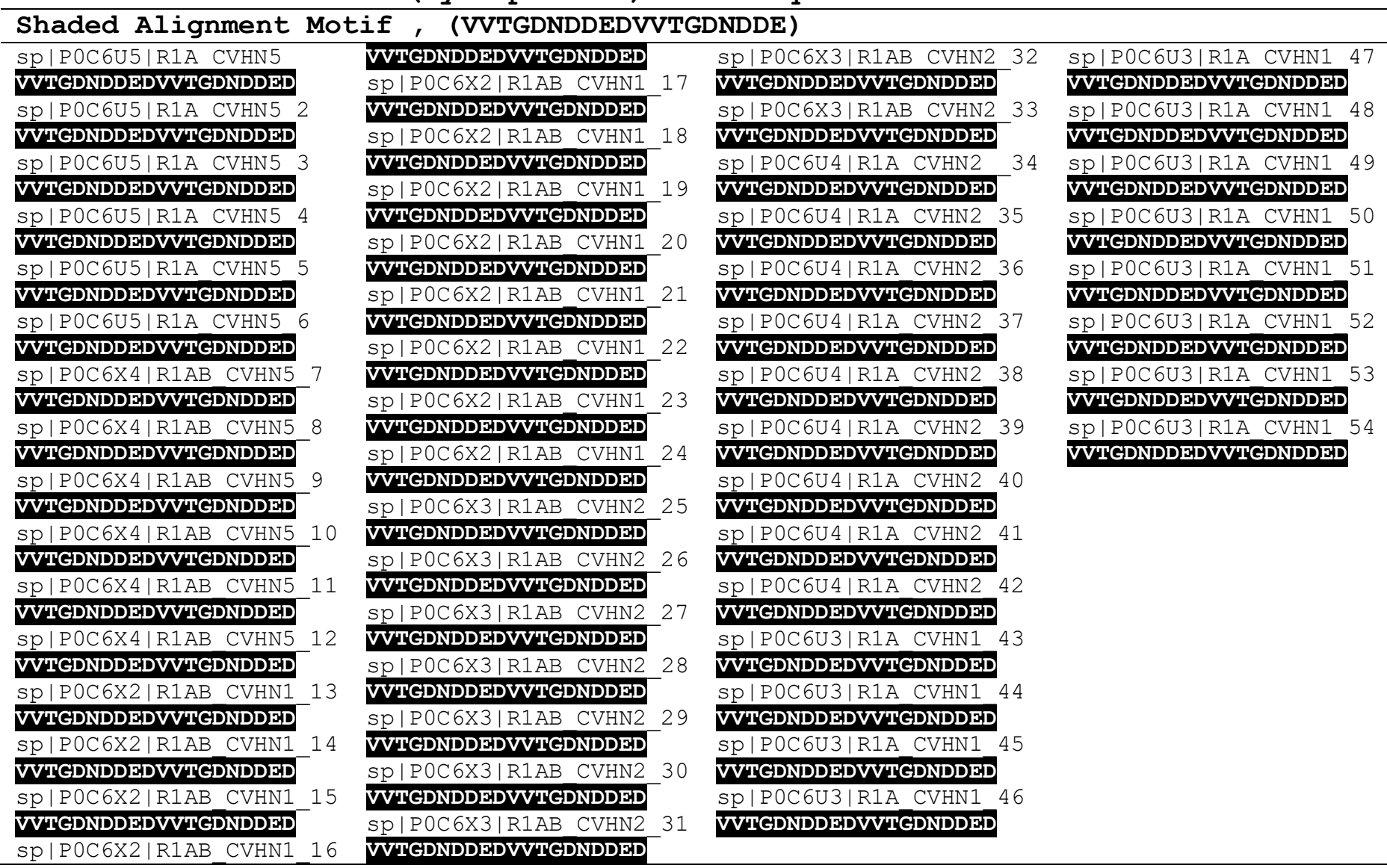

\subsection{Searching for the repeats by using YASS tool}

YASS uses seeds to detect potential similarity regions, and then tried to extend them to local alignments, this genomic search tool used multiple transition constrained spaced seeds that enable to search more fuzzy repeats, as non-coding DNA/RNA. The result of using YASS tool gave a dot plot graph then the zooming option was used to focus on the region of repeats. It took a diagonal repeats which was an indication for a repeated regions in the accession number (KF430201.1) of Human corona virus genome which aligned to itself as shown in the (Fig. 4a) and (Fig. 4b). The Zoom mode showed the repeated sequences as a Small Square (multiple diagonal lines paralleled to the main diagonal line of similarity) as shown in the (Fig. 4b). 


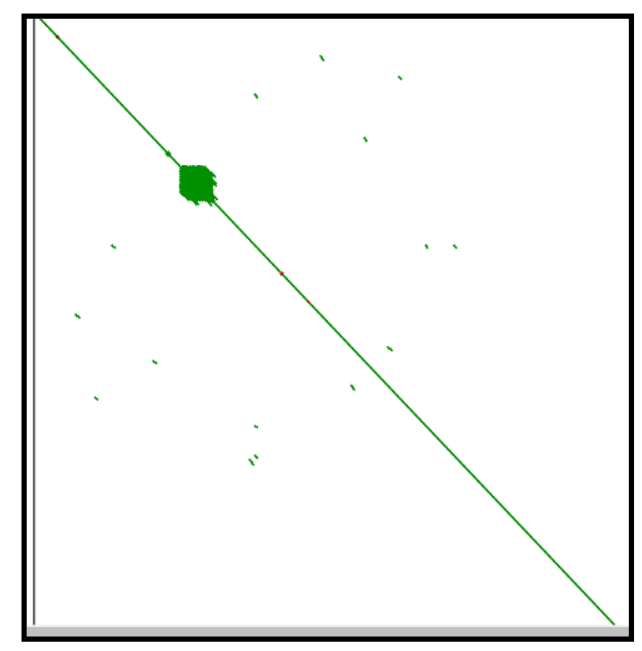

(Fig 4. a)

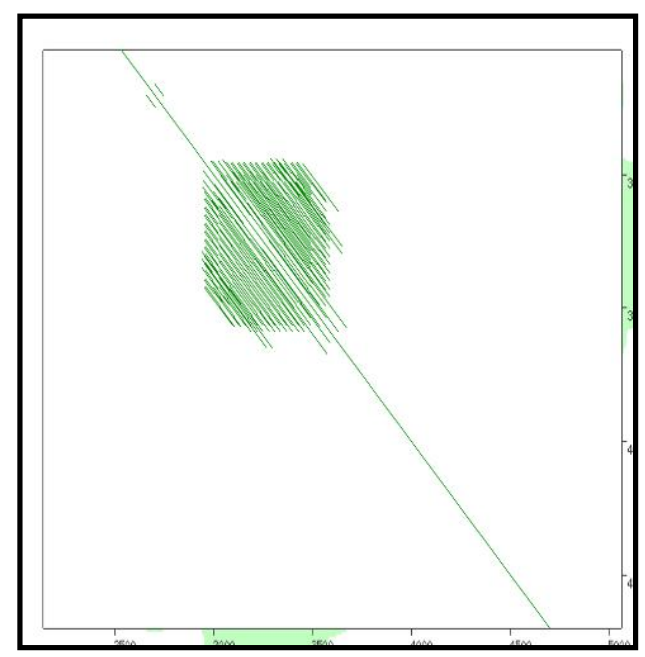

(Fig 4. b)

(Fig. 5 a) and (Fig. 5 b) shown the repeated sequences as a small square (multiple diagonal lines paralleled to the main diagonal line of similarity of the accession number (KF430201.1) that accession number was used as a model to ensure the sensitivity of RFRE tool to find repeat .

\subsection{The Possible recombination spot}

The RAT tool searched and expected the possible recombination spot, which occurred at nucleotide position 3012. This position shared and crossed among the twenty nine records of human corona virus as shown in (Fig. 5). and detected also as a start position of repeated sequences in the following accession numbers (KF686346.1, KF686344.1, KF686343.1, KF686342.1, KF686341.1 and KF686340.1) The possible recombination sites were produced and organized by the RAT tool in tabulated format as shown in the (Table 5). Deletion of part of the repeat region during the viral replication as a result of inexact replication by the recombination between the repeat regions of different Human coronavirus (HKU1) strains[2] 


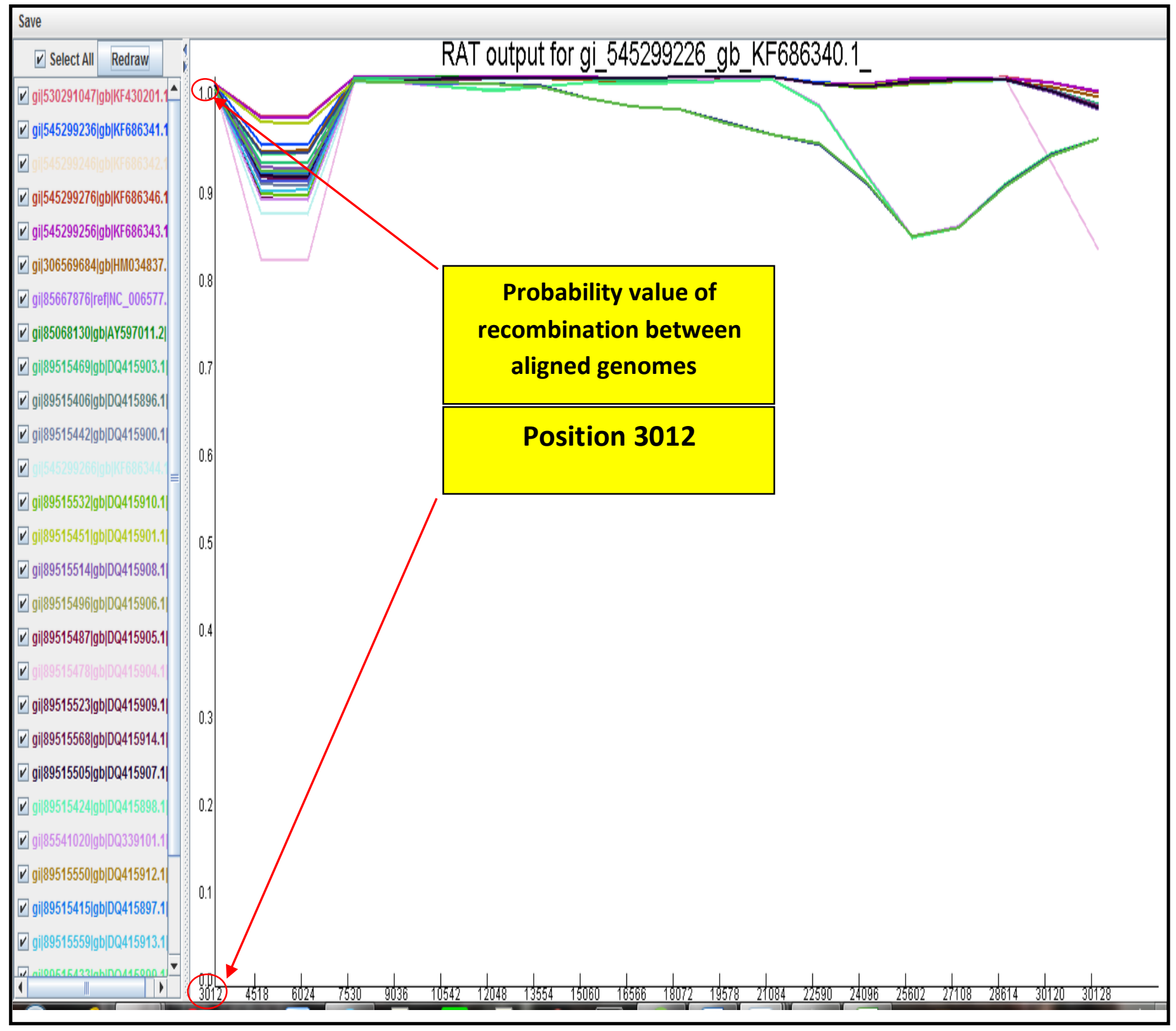

(Fig 5.) Shown the possible recombination spot occurs at position 3012. 
Table 5.0 Shown the possible recombination spot between every two accession numbers which occurred at position $\mathbf{3 0 1 2}$ among 29 records of human corona virus

\begin{tabular}{lc}
\hline Accession numbers & Position $\mathbf{3 0 1 2}$ and probabilities \\
\hline gi|530291047|gb|KF430201.1| & 0.9917 \\
gi|545299236|gb|KF686341.1| & 0.9917 \\
gi|545299246|gb|KF686342.1| & 0.991368 \\
gi|545299276|gb|KF686346.1| & 0.991368 \\
gi|545299256|gb|KF686343.1| & 0.991368 \\
gi|306569684|gb|HM034837.1| & 0.986056 \\
gi|85667876|ref|NC_006577.2| & 0.991036 \\
gi|85068130|gb|AY597011.2| & 0.991036 \\
gi|89515469|gb|DQ415903.1| & 0.990704 \\
gi|89515406|gb|DQ415896.1| & 0.990704 \\
gi|89515442|gb|DQ415900.1| & 0.990704 \\
gi|545299266|gb|KF686344.1| & 0.989376 \\
gi|89515532|gb|DQ415910.1| & 0.99004 \\
gi|89515451|gb|DQ415901.1| & 0.990704 \\
gi|89515514|gb|DQ415908.1| & 0.990372 \\
gi|89515496|gb|DQ415906.1| & 0.990372 \\
gi|89515487|gb|DQ415905.1| & 0.990704 \\
gi|89515478|gb|DQ415904.1| & 0.990372 \\
gi|89515523|gb|DQ415909.1| & 0.990372 \\
gi|89515568|gb|DQ415914.1| & 0.990372 \\
gi|89515505|gb|DQ415907.1| & 0.990704 \\
gi|89515424|gb|DQ415898.1| & 0.98506 \\
gi|85541020|gb|DQ339101.1| & 0.986056 \\
gi|89515550|gb|DQ415912.1| & 0.985724 \\
gi|89515415|gb|DQ415897.1| & 0.986056 \\
gi|89515559|gb|DQ415913.1| & 0.98506 \\
gi|89515433|gb|DQ415899.1| & 0.985392 \\
gi|85372431|gb|AY884001.1| & 0.985392 \\
gi|89515541|gb|DQ415911.1| & 0.986056 \\
gi|89515460|gb|DQ415902.1| & 0.986056 \\
\hline
\end{tabular}




\subsection{Assigning the detected repeats of Human corona virus (kf686343.1) on a graphical map}

The highest total length of the repeats $(480 \mathrm{bp})$ which found inside the completed genome of Human corona virus (kf686343.1) were assigned to the annotated map of accession number, (kf686343.1) by using Unipro UGENE ver. 1.16, 2015. The assigned repeats were located inside the pb1a gene (replicase polyprotien 1a) from the nucleotide position (3013-3492) as shown in (Fig 7.).

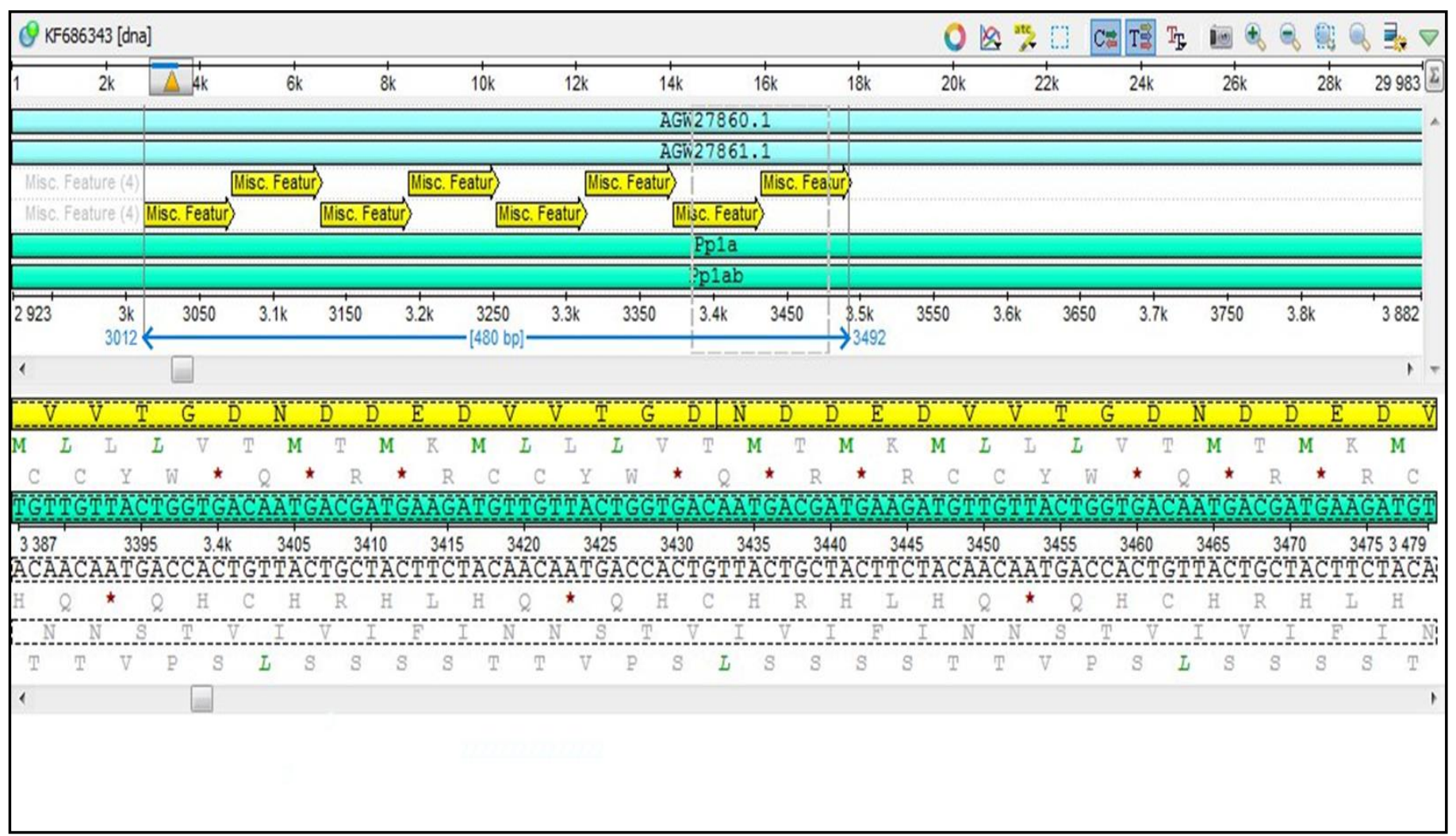

(Fig 7.) Shown the location of the extracted repeats from position (3012-3492) which annotated and added to genbank file of the accession number (kf686343.1) by using the UGENE ver. 1.16, annotation module. 


\section{Conclusions}

In conclusion, this study focused on the importance of identifying the tandem repeats in of human coronavirus (hku1) and make a relation between the genome size of (hku1) types and tandem repeats. The repeats which were found converted to amino acid sequence and used to build a conserved block of multiple sequence alignment to produce a specific motif for six sequences of CoV (HKU1) strains. Recombination between the repeated regions in the complete genomes of different CoV (HKU1) strains were observed in the recombination position (3012) which was also detected as a repeated position among the human corona virus and expected to be a key role of virus evolution. Maybe viruses combined partial genome sequences of hosts in infecting by using the repeats sequences as a hot spot region for recombination.

\section{Acknowledgement}

We thank Eng. Mohammed Tantawi for his guidance and advices during programming the tool

\section{References}

[1] Zhao, Zhixin, et al. "Genome-wide analysis of tandem repeats in plants and green algae." G3: Genes/ Genomes/ Genetics 4.1 (2014): 67-78.

[2] Woo, Patrick CY, et al. "Coronavirus diversity, phylogeny and interspecies jumping." Experimental Biology and Medicine 234.10 (2009): 1117-1127. 
[3] Lau, Susanna KP, et al. "Molecular epidemiology of human coronavirus OC43 reveals evolution of different genotypes over time and recent emergence of a novel genotype due to natural recombination." Journal of virology 85.21 (2011): 11325-11337.

[4] Aaziz, Rachid, and Mark Tepfer. "Recombination in RNA viruses and in virus-resistant transgenic plants." Journal of General Virology 80.6 (1999): 1339-1346.

[5] Jeffreys, Alec J., John Murray, and Rita Neumann. "High-resolution mapping of crossovers in human sperm defines a minisatellite-associated recombination hotspot." Molecular cell 2.2 (1998): 267-273.

[6] Bierne, Hélène, et al. "Isolation of a dnaE mutation which enhances RecA-independent homologous recombination in the Escherichia coli chromosome." Molecular microbiology 24.6 (1997): 1225-1234.

[7] Osterrieder, Nikolaus, Nina Wallaschek, and Benedikt B. Kaufer. "Herpesvirus genome integration into telomeric repeats of host cell chromosomes." Annual Review of Virology 1 (2014): 215-235.

[8] Biet, E., J-S. Sun, and M. Dutreix. "Conserved sequence preference in DNA binding among recombination proteins: an effect of ssDNA secondary structure." Nucleic acids research 27.2 (1999): 596-600.

[9] Zhao, Xiangyan, Yonglei Tian, Ronghua Yang, Haiping Feng, Qingjian Ouyang, You Tian, Zhongyang Tan et al. "Coevolution between simple sequence repeats (SSRs) and virus genome size." BMC genomics 13, no. 1 (2012): 435. 